\title{
Self-similar motion of laser half-space plasmas. I. Deflagration regime
}

\author{
Juan R. Sanmartín and A. Barrero \\ Escuela Técnica Superior de Ingenieros Aeronáuticos, Universidad Politécnica de Madrid, Madrid, Spain \\ (Received 14 June 1977; final manuscript received 18 July 1978)

\begin{abstract}
The one-dimensional self-similar motion of an initially cold, half-space plasma of electron density $n_{0}$, produced by the (anomalous) absorption of a laser pulse of irradiation $\phi=\phi_{0} t / \tau(0<t \leq \tau)$ at the critical density $n_{c}\left(n_{c} / n_{0} \equiv \epsilon \ll 1\right)$, is considered. The analysis allows for electron heat conduction and ion-electron energy exchange and retains three dimensionless numbers: $\epsilon, Z_{i}$ (ion charge number), and $\alpha=\left(9 \mathrm{k} / 4 \mathrm{~m}_{i}\right)$ $\left(\tau k^{2} n_{0}^{2} / \phi_{0} \bar{K}_{e}\right)^{2 / 3}$, where $k, m_{i}$ are Boltzmann's constant and the ion mass, and $\bar{K}_{e} \times$ (electron temperature $)^{5 / 2}=$ heat conductivity. If $\alpha \gg \epsilon^{-4 / 3}$, a deflagration wave separates an isentropic compression with a shock bounding the undisturbed plasma, and an isentropic expansion flow to the vacuum. The structures of these three regions are completely determined; in particular, the Chapman-Jouguet condition is proved and the density behind the deflagration is found. The deflagration-compression thickness ratio is large (small) for $\alpha \ll \epsilon^{-5 / 3}\left(\alpha>\epsilon^{-5 / 3}\right)$. The compression to expansion ratio for both energy and thickness is $O\left(\epsilon^{1 / 2}\right)$. For $Z_{i}$ large, a deflagration exists even if $\alpha \sim \epsilon^{-4 / 3}$. Condition $\alpha \gg \epsilon^{-4 / 3}$ may be applied to pulses that are not linear.
\end{abstract}

\section{INTRODUCTION}

In a recent paper ${ }^{1}$ the authors analyzed the self-similar motion generated in a plasma (initially cold and filling the entire space with uniform density $n_{0}$ ) by the absorption, in a given plane, of a laser pulse of irradiation linear in time, $\phi=\phi_{0} t / \tau(t \leqslant \tau)$. The results showed, in detail, the importance of heat conduction and electronion temperature relaxation against convection of energy, and their dependence on a single parameter $\alpha \propto\left(n_{0}^{2} \tau /\right.$ $\left.\phi_{0}\right)^{2 / 3}$. The existence of self-similar motion in a plasma with heat conduction and different ion and electron temperatures was noticed by Anisimov. ${ }^{2}$ A broad discussion of conditions for self-similar plasma motion has recently been given. ${ }^{3}$

Laser fusion requires compressing a DT pellet to very high densities, heating only its core. ${ }^{4,5}$ The radiation is usually absorbed at electron densities close to the critical value, $n_{c}$ (much less than $n_{0}$ ) inside a hot, rarefied corona ablated by the laser. Hydrodynamics is an essential part of the phenomenon.

In this work we have considered an extension of Ref. 1: The plasma initially occupies a half-space and the laser radiation is assumed absorbed uniformly in the plane where the electron density equals $n_{c}$. Clearly, there are now two parameters, $n_{c} / n_{0}$ and $\alpha$; moreover, there is also a new effect, the corona expansion to the vacuum, competing with ion-electron relaxation and energy conduction and convection. The analysis, therefore, includes the essential hydrodynamics of laser fusion, and in general, of overdense laser plasmas. The limitation to one-dimensional situations and linear pulses (for which a self-similar solution is possible) implies no restriction, since our purpose is to clearly establish the general dependence of the motion on the laser parameters (accordingly, nonhydrodynamic effects such as radiation pressure and emission, or nuclear fusion, have been omitted in the analysis). Actually, some quantitative results obtained here (the structure of deflagration layers in overdense laser plasmas) do remain valid for very general situations.

We have found that for $\epsilon \equiv n_{c} / n_{0}$ small (overdense plas ma) there are three main flow regimes: $\alpha \ll 1,1 \ll \alpha$ $\ll \epsilon^{-4 / 3}$, and $\epsilon^{-4 / 3} \ll \alpha$, (with transition regimes at $\alpha \sim 1$ and $\left.\alpha \sim \epsilon^{-4 / 3}\right)$. The first two regimes have been considered in a companion paper ${ }^{6}$; here, we limit ourselves to the case $\alpha \gg \epsilon^{-4 / 3}$ [more precisely $\left(\alpha \epsilon^{4 / 3}\right)^{3 / 2} \gg 1$ ] which seems to correspond to the deflagration regime (not too high $\phi_{0}$ ) previously discussed in the literature.

Deflagration waves in overdense plasmas were first studied in detail by Fauquignon and Floux ${ }^{7}$ for constant irradiation; their analys is was extended to include the structure of the deflagration layer by Bobin. ${ }^{8}$ Two-dimensional and spherical effects on the deflagration have been considered by Pert, ${ }^{9}$ and Gitomer et al. ${ }^{10}$ respectively. The expansion of a plasma into a vacuum without energy absorption was considered by Gurevitch et $a l .^{11}$ and Crow et al. ${ }^{12}$

As expected, three different flow regions ${ }^{7}$ emerge naturally from our asymptotic analysis for $\epsilon \ll<1$ and $\left(\alpha \epsilon^{4 / 3}\right)^{3 / 2} \gg 1$ : a region of shocked material moving isentropically into the undisturbed cold medium, a plasma deflagration layer where radiation is absorbed and heat conduction and ion-electron energy exchange are important, and a region of low density plasma expanding into the vacuum. Figure 1 shows an overall picture of the motion obtained from the asymptotic results for those three regions, in a typical laser plasma [Nd glass laser, $\phi_{0}=5 \times 10^{12} \mathrm{~W} \mathrm{~cm}^{-2}, \tau=70 \mathrm{nsec}$, deuterium at solid density: $n_{0} \simeq 5 \times 10^{22} \mathrm{~cm}^{-3}$, for which $\epsilon \simeq 1 / 50,\left(\alpha \epsilon^{4 / 3}\right)^{3 / 2}$ $\simeq 10.4]$; the excellent matching between the solutions for the different regions confirms the validity of the asymptotic analysis.

In many practical applications, the assumption that the material in the isentropic compression region behind the shock is a plasma, may not be valid (clearly, this 


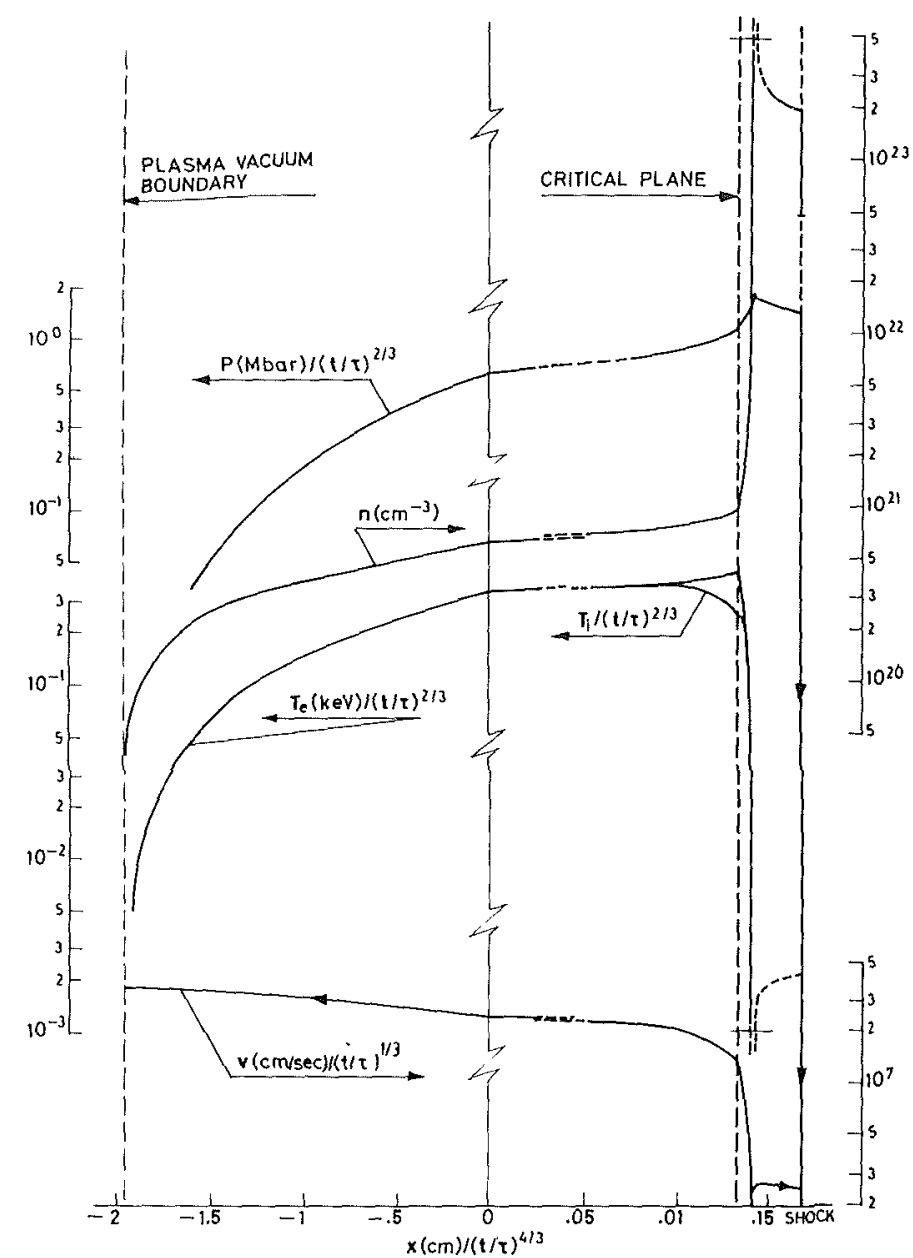

FIG. 1. Overall picture of density, velocity, pressure, and ion and electron temperatures, obtained from asymptotic results for the three flow regions (Figs. 2,4 , and 6 ), in the case of a Nd laser pulse $\phi=\phi_{0} t / \tau\left(\phi_{0}=5 \times 10^{12} \mathrm{~W} \mathrm{~cm}^{-2}, \tau=70 \mathrm{nsec}\right)$ incident on a solid deuterium half-space; notice the scale change at $x=0$. Temperature in the region behind the shock, and its minimum, are very rough approximations for the conditions of the figure since then the medium is not a plasma there; correct values could be obtained from an appropriate equation of state.

is the case for the conditions of Fig. 1). Nonetheless, for a hydrogen isotope, such as deuterium, in the density-pressure range of $F i g .1$, results for the compression region, obtained assuming the medium there to be a plasma, (results shown in the figure) are very accurate, as we shall see, except for temperature and too large densities (as in the thin transition layer separating deflagration from compression region). In addition, we notice that the results for the deflagration layer and the expansion flow are entirely independent of conditions in the compression region, except for the deflagration speed (in Fig. 1, the position of the right boundary of the deflagration layer).

Some results obtained here, which are either new or corrections to results found in the literature, are:

(i) A deflagration exists if, and only if,

$\left(\alpha \epsilon^{4 / 3}\right)^{3 / 2} \equiv 2.10 \times 10^{-30} \frac{Z_{i}}{b\left(Z_{i}\right)} \frac{\tau(\mathrm{nsec})\left[n_{c}\left(\mathrm{~cm}^{-3}\right)\right]^{2}}{A_{i}^{3 / 2} \phi_{0}\left(\mathrm{~W} \mathrm{~cm}^{-2}\right)} \gg 1$.
This criterion involves the three main laser parameters, maximum irradiation $\phi_{0}$, pulse duration $\tau$, and frequency $\omega$

$$
\omega \equiv\left(4 \pi e^{2} n_{c} / m_{e}\right)^{1 / 2},
$$

( $e$ and $m_{e}$ are the electron charge and mass) together with the ion mass and charge numbers $\left(A_{i}\right.$ and $\left.Z_{i}\right)$ of the plasma; $b\left(Z_{i}\right)$ is given by

$$
b \equiv Z_{i} \bar{K}_{e}\left(Z_{i}\right) / \bar{K}_{e}(1) \text {, }
$$

where $\bar{K}_{e}$ is given by Spitzer $\left(K_{e} \equiv \bar{K}_{e} T_{e}^{5 / 2}\right.$ and $T_{e}$ are the electron heat conductivity and temperature, respectively). ${ }^{13}$ For pulses $\phi(t)$ that are not linear, either $\phi(t) / t$ or $d \phi / d t$ (whichever is the larger) should be used instead of $\phi_{0} / \tau$ in (1); clearly, for some pulse shapes $\alpha$ will be ill-defined. Notice that condition (1) cannot be satisfied with a $\mathrm{CO}_{2}$ laser except for long pulses of low irradiation.

(ii) The structure of the deflagration layer is rigorously determined; in particular, electron heat conduction and ion-electron energy exchange are retained simultaneously, and the position of the critical plane is self-consistently determined (heat flows from this plane toward the rarefaction). ${ }^{8}$ The density just behind the deflagration is neither $n_{c}{ }^{7,8}$ nor $n_{c} \times$ (specific heat ratio $)^{-1 / 2},{ }^{9}$ but depends on $Z_{i}$.

(iii) The expansion is isentropic, not isothermal, a point not well established until now ${ }^{7-9}$; thus, temperatures drop to zero in the plasma-vacuum boundary ${ }^{10}$ (a look at the numerical results by Mulser ${ }^{14}$ and Cooper ${ }^{15}$ provides evidence for this point). Isothermal expansions found in numerical simulations of laser fusion, ${ }^{16,17} \mathrm{cor}-$ respond to the regimes $\alpha \ll \epsilon^{-4 / 3}$, for which we do find an isothermal expansion ${ }^{6}$ (but no deflagration).

(iv) The deflagration is thinner or thicker than the compression region for $\alpha \epsilon^{-5 / 3}$ large or small, respectively; for $\alpha \epsilon^{-5 / 3}=O(1)$, as in Fig. 1, both regions have comparable size. Thick and thin deflagrations in relation to two-dimensional effects have been discussed by Pert. ${ }^{g}$

In Sec. II we establish the self-similar variables and equations, and discuss the equations to be used in the compression region. Sections III $-\mathrm{V}$ contain the mathematical analysis of the three main flow regions. A summary and discussion of the results is given in Sec. VI. The transition layer is briefly considered in the Appendix.

\section{GENERAL EQUATIONS}

The evolution of the plasma is governed by the equations

$$
\begin{aligned}
& \frac{D n}{D t}=-n \frac{\partial v}{\partial x}, \quad\left(\frac{D}{D t} \equiv \frac{\partial}{\partial t}+v \frac{\partial}{\partial x}\right), \\
& m_{i} n \frac{D v}{D t}=-\frac{\partial}{\partial x}\left[n k\left(Z_{i} T_{e}+T_{i}\right)\right], \\
& n T_{e} \frac{D}{D t}\left(k \ln \frac{T_{e}^{3 / 2}}{n}\right)= \frac{\partial}{\partial x}\left(K_{e} \frac{\partial T_{e}}{\partial x}\right)-\frac{3}{2} k n \frac{T_{e}-T_{i}}{t_{e i}} \\
&+\phi(t) \delta\left(x-x_{c}\right),
\end{aligned}
$$




$$
\frac{n}{Z_{i}} T_{i} \frac{D}{D t}\left(k \ln \frac{T_{i}^{3 / 2}}{n}\right)=\frac{3}{2} k n \frac{T_{e}-T_{i}}{t_{e i}}
$$

$x_{c}$ being such that

$$
n\left(x_{c}\right)=n_{c} \text {. }
$$

The ion-electron energy relaxation time is ${ }^{13} t_{e i}$ $=\bar{t}_{e i} T_{e}^{3 / 2} n^{-1}$; we have assumed both quasi-neutrality $\left(n_{e}\right.$ $\simeq Z_{i} n_{i} \equiv n$ ) and near Maxwellian distribution functions. [For a discussion of Eqs. (3a)-(3d), see Ref. 1.] The half-space $x>0$ is that initially occupied by the plasma; $n, v, T$, and $m$ are density, velocity, temperature, and mass, $k$ is Boltzmann's constant, $\delta$ is Dirac's function. Subscripts $e, i$ refer to electrons and ions.

The laser pulse starts at $t=0$ when the plasma is cold and at rest

$n=n_{0}, \quad v=T_{e}=T_{i}=0 \quad(x \geqslant 0) ; \quad n=0 \quad(x<0)$.

The boundary conditions are: For any $t$ and $x=\infty$

$$
n=n_{0}, \quad v=T_{e}=T_{i}=0 ;
$$

for $x=x_{v}(t)$ (the plasma-vacuum boundary on the left)

$$
n=0, \quad v=d x_{v} / d t .
$$

A condition on $T_{e}$ should be given at $x_{v}$ if heat conduction is important there; if the plasma remains collisiondominated near $x_{v}$, we should have $T_{e}\left(x_{v}, t\right)=0$ since the electron mean-free-path is proportional to $T_{e}^{2} n^{-1}$.

Taking as constants the Coulomb logarithms in $\bar{K}_{e}$ and $\bar{t}_{e i}$, the motion of the plasma is self-similar if $\phi(t)$ is linear,

$$
\phi=\phi_{0} t / \tau, \quad 0<t \leqslant \tau .
$$

Then defining

$$
\xi=x\left[(t / \tau)^{4 / 3} 3 v_{0} \tau / 4\right]^{-1}, \quad \bar{n}(\xi)=n n_{0}^{-1},
$$

$u(\xi)=v\left[(t / \tau)^{1 / 3} v_{0}\right]^{-1}, \quad \theta_{j}(\xi)=T_{j}\left[(t / \tau)^{2 / 3} T_{0}\right]^{-1} \quad(j=e, i)$,

where

$$
v_{0}=\frac{4}{3}\left(\frac{\bar{K}_{e}^{2} \phi_{0}^{5}}{k^{7} n_{0}^{7} \tau^{2}}\right)^{1 / 9}, \quad T_{0}=\left(\frac{\phi_{0}^{2} \tau}{k n_{0} \bar{K}_{e}}\right)^{2 / 9}
$$

and introducing

$$
\alpha=\left(9 k / 4 m_{i}\right)\left(\tau k^{2} n_{0}^{2} / \phi_{0} \bar{K}_{e}\right)^{2 / 3},
$$

Eqs. (3)-(5) become

$$
\begin{aligned}
& \frac{d \bar{n}}{d \xi}=\frac{\bar{n}}{\xi-u} \frac{d u}{d \xi}, \\
& u-4(\xi-u) \frac{d u}{d \xi}=-\frac{\alpha}{\bar{n}} \frac{d}{d \xi}\left[\bar{n}\left(Z_{i} \theta_{e}+\theta_{i}\right)\right], \\
& \bar{n}\left[\theta_{e}\left(1+\frac{4}{3} \frac{d u}{d \xi}\right)-2(\xi-u) \frac{d \theta_{e}}{d \xi}\right] \\
& \quad=\frac{d}{d \xi} \theta_{e}^{5 / 2} \frac{d \theta_{e}}{d \xi}-4.3 b \alpha \bar{n}^{2} \frac{\theta_{e}-\theta_{i}}{\theta_{e}^{3 / 2}}+\delta\left(\xi-\xi_{c}\right), \\
& \frac{\bar{n}}{Z_{i}}\left[\theta_{i}\left(1+\frac{4}{3} \frac{d u}{d \xi}\right)-2(\xi-u) \frac{d \theta_{i}}{d \xi}\right]=4.3 b \alpha \bar{n}^{2} \frac{\theta_{e}-\theta_{i}}{\theta_{e}^{3 / 2}}, \\
& \bar{n}\left(\xi_{c}\right)=n_{c} / n_{0} \equiv \epsilon,
\end{aligned}
$$

$$
\begin{array}{ll}
\bar{n}(\infty)=1, & u(\infty)=\theta_{e}(\infty)=\theta_{i}(\infty)=0, \\
\bar{n}\left(\xi_{v}\right)=0, & u\left(\xi_{v}\right)=\xi_{v} \quad\left[\theta_{e}\left(\xi_{v}\right)=0\right] .
\end{array}
$$

Setting all Coulomb logarithms arbitrarily equal to eight, we have

$$
\begin{aligned}
& v_{0}(\mathrm{~cm} / \mathrm{sec}) \simeq 1.24 \times 10^{17}\left(b / Z_{i}\right)^{2 / 9} \phi_{0}^{5 / 9} \tau^{-2 / 9} n_{0}^{-7 / 9}, \\
& T_{0}(\mathrm{keV}) \simeq 6.87 \times 10^{-2}\left(Z_{i} / b\right)^{2 / 9} \phi_{0}^{4 / 9} \tau^{2 / 9} n_{0}^{-2 / 9}, \\
& \alpha \simeq 1.64 \times 10^{-20}\left(Z_{i} / b\right)^{2 / 3} \phi_{0}^{-2 / 3} \tau^{2 / 3} n_{0}^{4 / 3} A_{i}^{-1},
\end{aligned}
$$

where $\phi_{0}, \tau$, and $n_{0}$ are measured in $\mathrm{W} \mathrm{cm}^{-2}$, nsec, and $\mathrm{cm}^{-3}$, respectively; $b$ ranges from 1 for $Z_{i}=1$, to 4.2 for $Z_{i}=\infty$ 。

Now, while we assumed from the beginning that the medium was everywhere a plasma, it would be important to consider having at $t=0$ a condensed phase of matter (e.g., solid deuterium); in this case, the material within the is entropic compression region which is found to develop adjoining the undisturbed medium cannot be assumed to be a plasma, and there the validity of the preceding equations should be questioned. Fortunately, a self-similar solution exists for the isentropic flow if, for fixed density, the specific energy $E$ is proportional to the pressure $P$. This condition (much weaker than that requiring the medium to be a plasma) is approximately satisfied ${ }^{18}$ by deuterium for pressures (of the order of $10^{6} \mathrm{~atm}$ ) and densities (not far above solid density), obtained in a typical situation (Fig. 1). The deuterium is then in a fluid phase, and the cold (or elastic) pressure and specific energy are negligible because of the very low bulk modulus of deuterium, ${ }^{19}$ while the Thomas-Fermi-Dirac limit state is far from being reached. The argument also applies to hydrogen or tritium.

In an isentropic region, heat conduction should be negligible, while $\theta_{i} \simeq \theta_{e} \equiv \theta$; then, addition of Eqs. (11a) and $(11 \mathrm{~b})$ leads to

$$
\theta\left(1+\frac{4}{3} \frac{d u}{d \xi}\right)-2(\xi-u) \frac{d \theta}{d \xi}=0
$$

If we now use $P$ (instead of $T$ ) together with $n$ and $v$ to describe the flow, and write

$$
P \equiv\left(n_{0} / Z_{i}\right) k T_{0}(t / \tau)^{2 / 3} \bar{P}(\xi)
$$

Eqs. (10) and (14) become

$$
\begin{aligned}
& u-4(\xi-u) \frac{d u}{d \xi}=-\frac{\alpha}{\bar{n}} \frac{d \bar{P}}{d \xi}, \\
& \bar{P}\left(1+\frac{10}{3} \frac{d u}{d \xi}\right)-2(\xi-u) \frac{d \bar{P}}{d \xi}=0,
\end{aligned}
$$

where

$$
\bar{P}=\left(Z_{i}+1\right) \bar{n} \theta \text {. }
$$

Now, if we do not have a plasma but do have $E \propto P$, we introduce a Gruneisen-like coefficient

$$
\Gamma(n)=P / E m_{i} n \quad\left(Z_{i}=1\right),
$$

and using (15) in the equation for an isentropic evolution

$$
\frac{D E}{D t}+P \frac{D}{D t} \frac{1}{m_{i} n}=0 \text {, }
$$


arrive at

$$
\bar{P}\left[1+2\left(1+\Gamma+\frac{d \ln \Gamma}{d \ln \bar{n}}\right) \frac{d u}{d \xi}\right]-2(\xi-u) \frac{d \bar{P}}{d \xi}=0 .
$$

If $\Gamma=2 / 3$, as in a plasma, $\mathrm{Eq} .\left(14^{\prime}\right)$ is recovered from $\left(14^{\prime \prime}\right)$. Notice that Eqs. $(9)$ and $\left({ }^{\prime} 0^{\prime}\right)$ remain valid although (16) does not. We have avoided using temperature as a variable because a simple equation of state may be lacking, as for deuterium in the conditions mentioned (dissociation, electronic excitation, etc., are then important). Appropriate values of $\Gamma+d \ln \Gamma / d \ln \bar{n}$ are not far from unity, and the results, as we shall see, are practically equal to those obtained when a value of $\frac{2}{3}$ is taken; thus, we may still consider the medium in the compression region to be a plasma ${ }^{14}$ [except for the fact that temperature is not given by Eq. (16), but should be taken from appropriate tables ${ }^{18}$ once $\bar{P}(\xi)$ and $\bar{n}(\xi)$ are found]. We emphasize that this conclusion would be valid for deuterium-tritium.

\section{ISENTROPIC COMPRESSION REGION}

For $\alpha$ large we expect that, as in Ref. 1, the disturbance front will be a shock, which precedes a region of isentropic compression. Rewriting Eqs. (9), (10), and the sum of (11a) and (11b), in the form

$\bar{n}=\frac{d}{d \xi}[\bar{n}(\xi-u)]$,

$5 \bar{n} u=\frac{d}{d \xi}\left[4 \bar{n} u(\xi-u)-\alpha \bar{n}\left(Z_{i} \theta_{e}+\theta_{i}\right)\right]$,

$$
\begin{gathered}
3 \bar{n}\left(Z_{i} \theta_{e}+\theta_{i}+\frac{4 u^{2}}{3 \alpha}\right)=\frac{d}{d \xi}\left[2 \bar{n}(\xi-u)\left(Z_{i} \theta_{e}+\theta_{i}+\frac{4 u^{2}}{3 \alpha}\right)\right. \\
\left.-\frac{4}{3} \bar{n} u\left(Z_{i} \theta_{e}+\theta_{i}\right)+Z_{i} \theta_{e}^{5 / 2} \frac{d \theta_{e}}{d \xi}\right]+Z_{i} \delta\left(\xi-\xi_{c}\right),
\end{gathered}
$$

and integrating across the shock, we obtain the jump conditions (for a plasma)

$$
\bar{n}_{f}=4, \quad u_{f}=3 \xi_{f} / 4, \quad \theta_{f}=3 \xi_{f}^{2}\left[4\left(Z_{i}+1\right) \alpha\right]^{-1} ;
$$

$f$ labels the values just behind the shock. Integration of Eq. (18c) between $\xi_{v}$ and $\xi_{f}$ leads to $\xi_{f}=\gamma \alpha^{1 / 3}$, where $\gamma$ is an unknown constant (determining the shock position) which cannot be large.

Now introducing the variables

$\eta=\xi / \xi_{f}, \quad \nu=\bar{n} / \bar{n}_{f}, \quad y=u / u_{f}, \quad z_{j}=\theta_{j} / \theta_{f}$,

into Eqs. (9)-(11) one verifies that heat conduction is $O\left(\gamma^{3} \alpha^{-3 / 2}\right)$ and thus may be neglected, while the ion-electron relaxation is $O\left(\alpha^{3 / 2} \gamma^{-3}\right)$, so that the subscript $j$ may be suppressed in (20). Then, Eqs. (9), (10), and (14) become

$$
\begin{aligned}
& \frac{d \nu}{d \eta}=\frac{3 \nu}{4 \eta-3 y} \frac{d y}{d \eta}, \\
& y-(4 \eta-3 y) \frac{d y}{d \eta}=-\frac{1}{\nu} \frac{d}{d \eta}(\nu z), \\
& 2 z(1+d y / d \eta)-(4 \eta-3 y) d z / d \eta=0 ;
\end{aligned}
$$

this system, with boundary conditions

$$
\nu(1)=y(1)=z(1)=1,
$$

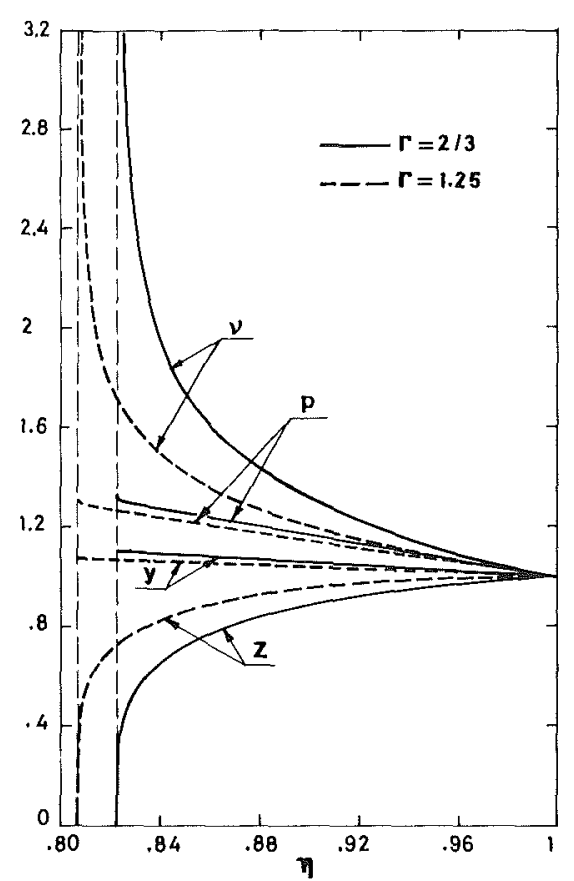

FrG. 2。 Density, $\nu$, velocity, $y$, pressure, $p$, and temperature, $z$, vs distance, $\eta$, within the isentropic compression region for $\Gamma=2 / 3$ (plasma) and $\Gamma=1.25$ ( $\Gamma \equiv$ ratio of pressure to internal energy per unit volume); the variables have been normalized with the values behind the shock.

has been solved in Ref. 1. Numerical results for $\nu, y$, $z$, and $p=\bar{P} / \bar{P}_{f}\left(\bar{P}_{f}=3 \xi_{f}^{2} \alpha^{-1}\right)$ are shown in Fig. 2. In the neighborhood of a value $\bar{\eta} \simeq 0.82$, the solution behaves as $y \simeq \frac{4}{3} \bar{\eta}-\frac{2}{5}(\eta-\bar{\eta}), \quad z \simeq B_{1}(\eta-\bar{\eta})^{3 / 13}, \quad \nu \simeq B_{2}(\eta-\bar{\eta})^{-3 / 13}$

where $B_{1} \simeq 1.70, B_{2} \simeq 0.78$. The expression $\nu(4 \eta-3 y)$ (mass flow relative to the moving plane $\eta$ ) goes to zero as $\eta$ approaches $\bar{\eta}$ from the right. Equation (18a) shows, however, that $\bar{n}(\xi-u)$ must grow monotonically rightward from a zero value at $\xi_{v}$; thus, $4 \eta-3 y$ should be positive everywhere. Clearly, the is entropic compression solution ceases to be valid near $\bar{\eta}$.

We shall now confirm that the preceding results are also valid in some situations for which the medium is not a plasma. First of all, the density ratio across a strong shock in an arbitrary medium is

$$
\bar{n}_{f} \equiv n_{f} / n_{0}=1+\left(2 E m_{i} n / P\right)_{f} ;
$$

if, in the pressure range of interest, $P / m_{i} n E(P, n) \simeq 2 / 3$ for $n \simeq 4 n_{0}$, we would still have $n_{f} \simeq 4, u_{f} \simeq 3 \xi_{f} / 4, \bar{P}_{f}$ $\simeq 3 \xi_{f}^{2} \alpha^{-1}$. Then using $\eta, \nu, y$, and $p$ as defined previously, Eqs. (21) and (22) remain unchanged while the energy equation reads

$$
p\left[1+\frac{3}{2}\left(1+\Gamma+\frac{d \ln \Gamma}{d \ln \nu}\right) \frac{d y}{d \eta}\right]-\frac{1}{2}(4 \eta-3 y) \frac{d p}{d \eta}=0,
$$

where we assumed $\Gamma \equiv P / m_{i} n E$ nearly independent of $P$; for a plasma $\Gamma=2 / 3$ and Eq. (23) is recovered from (26) and $p=\nu z$. We have solved Eqs. (26), (21), and (22) with $\nu z=p$ for $\Gamma=1.25$; the results are shown in Fig. 2. Clearly, pressure, velocity, and position of 
$\bar{\eta}$ depend extremely weakly on $\Gamma$ (and therefore on its dependence on density), and, in this respect, we may set $\Gamma=2 / 3$; density would be then described in a reasonably correct way. Notice that $z=p / \nu$ need not be proportional to temperature so that results for $z$ are limited to the plasma case. The conditions on $\Gamma$ are satisfied by a monatomic ideal gas, and by hydrogen isotopes with $P$ in the mega-bar range, and $n$ not far above solid density.

\section{ISENTROPIC EXPANSION REGION}

We shall now make the ansatz that the plasma expands (moving leftward) all through the interval between $\eta_{v}$ and $\bar{\eta}$, the density there being of the order of the critical density

$$
\nu \sim \bar{n}_{c} / \bar{n}_{f}=O(\epsilon) .
$$

To match the isentropic compression solution, the density should then increase from zero at $\eta_{v}$ to infinity at $\bar{\eta}$, while the temperature should vanish at both $\eta_{v}$ and $\bar{\eta}$, having a maximum in between. As in Ref. 1 a very thin transition layer of dense, cold plasma, where $\nu$ peaks, $z$ bottoms out, and $y$ changes sign, should exist between the expansion and compression regions. We defer a dis cussion of this layer, which affects the solution outside it negligibly to the Appendix.

Writing Eq. (18b) in the variables defined in (20) and integrating across the transition layer, it is shown that in the expansion region, we have

$$
z=O\left(\epsilon^{-1}\right) \text {. }
$$

Further, we notice that the compression solution satisfies

$$
\int_{\ddot{\eta}}^{1} 5 \nu y d \eta=B_{1} B_{2}
$$

and thus momentum conservation leads to $\int_{\eta_{\nu}}^{\bar{\eta}} 5 \nu y d \eta$ $=O(1) ;$ since conditions at $\xi_{v}$ show that $y / \eta=O(1)$, we arrive at $\nu y^{2}=O(1)$ or

$$
y=O\left(\epsilon^{-1 / 2}\right), \quad \eta=O\left(\epsilon^{-1 / 2}\right) .
$$

Thus, using appropriately scaled variables we find that $\eta$ is negative throughout the expanding plasma, the interval $0<\eta<\bar{\eta}$ being of negligible extent. Similarly, one may set $y(0) \simeq y\left(\bar{\eta}^{-}\right) \simeq 0$, since $y\left(\bar{\eta}^{+}\right)=O(1)$; moreover, $y<0$ in the entire expansion, as assumed, because $(4 \eta-3 y)$ must be positive throughout the plasma (see end of last section). Finally, the energy in the compressed region comes out to be a small fraction of the total energy in the plasma; integration of $\mathrm{Eq}$. (18c) leads directly to $\gamma=\bar{\gamma} \epsilon^{1 / 6}$, where $\bar{\gamma}$ is an inknown constant of order unity.

Introducing variables scaled according to (27)-(29), into Eqs. (9)-(11), we find that the conduction term is $O\left(\alpha^{-3 / 2} \epsilon^{-2}\right)$ as compared with convection terms of order unity, while the ion-electron energy relaxation term is $O\left(\alpha^{3 / 2} \epsilon^{2}\right)$. Thus, if $\alpha \gg \epsilon^{-4 / 3}$, the expansion region would be isentropic. However, since electron heat conduction must be important near the critical density plane, a conduction layer, necessarily thin compared with the size given in (29), should exist within the expansion region, lying between the isentropic expansion flow on the left and $\bar{\eta}$. The structure of this layer, which may be considered as a deflagration wave because the temperature and velocity increase, and the density and pressure decrease across it, will be determined in the next section.

Clearly, if $\alpha=O\left(\epsilon^{-4 / 3}\right)$, the isentropic expansion and the deflagration layer merge into each other, and the transport and relaxation terms become important throughout the expansion flow. Thus, there is no deflagration unless $\alpha \gg \epsilon^{-4 / 3}$.

Now, let $\nu_{d}, z_{d}$, and $y_{d}$ be the unknown values of $\nu$, $z$, and $y$ at the base of the isentropic expansion (just behind the deflagration), assumed of order $\epsilon, \epsilon^{-1}$ and $\epsilon^{-1 / 2}$, respectively; then defining

$\hat{\eta}=\eta /\left(-y_{d}\right), \quad \hat{y}=y /\left(-y_{d}^{\prime}\right), \quad \hat{\nu}=\nu / \nu_{d}, \quad \hat{z}=z / z_{d}$,

Eqs. (21)-(23) directly yield

$$
\begin{aligned}
& \frac{d \hat{\nu}}{d \hat{\eta}}=\frac{3 \hat{\nu}}{4 \hat{\eta}-3 \hat{y}} \frac{d \hat{y}}{d \hat{\eta}}, \\
& \hat{\nu}[\hat{y}-(4 \hat{\eta}-3 \hat{y}) d \hat{y} / d \hat{\eta}]=-H d(\hat{\nu} \hat{z}) / d \hat{\eta}, \\
& 2 \hat{z}(1+d \hat{y} / d \hat{\eta})-(4 \hat{\eta}-3 \hat{y}) d \hat{z} / d \hat{\eta}=0,
\end{aligned}
$$

where $H=z_{d} / y_{d}^{2}$. As boundary conditions we have

$$
\begin{aligned}
& \hat{\nu}=\hat{z}=-\hat{y}=1, \quad \text { at } \hat{\eta}=0 \\
& \hat{v}=\hat{z}=0, \quad \hat{v}_{v}=4 \hat{\eta}_{v} / 3, \text { at } \hat{\eta}=\hat{\eta}_{v} .
\end{aligned}
$$

A first integral of the system $(31)-(33)$ is the usual adiabatic integral of self-similar isentropic flow, ${ }^{20}$

$$
\hat{\nu}^{7 / 3}(4 \hat{\eta}-3 \hat{y}) \hat{z}^{-2}=3 \text {. }
$$

It is easy to see that if $\hat{\nu}\left(\hat{\eta}_{v}\right)=0$, condition $\hat{z}\left(\hat{\eta}_{v}\right)=0$ follows from (35) and need not be included as a boundary condition; that is, the isentropic expansion is indeed collision dominated [see comment following Eq. (5c)]. Then, we must solve Eq. (33) and a combination of (31) and (32)

$$
\hat{y}-(4 \hat{\eta}-3 \hat{y}) \frac{d \hat{y}}{d \hat{\eta}}=-H\left(\frac{d \hat{z}}{d \hat{\eta}}+\frac{3 \hat{z}}{4 \hat{\eta}-3 \hat{y}} \frac{d \hat{y}}{d \hat{\eta}}\right),
$$

for $\hat{y}$ and $\hat{z}$. Now defining

$$
\hat{Y}=\hat{y} / \hat{\eta}, \quad \hat{Z}=H \hat{z} / \hat{\eta}^{2},
$$

we arrive at a phase-space equation similar to that analyzed in Ref. 1 for the compression region

$$
\frac{d \hat{Z}}{d \hat{Y}}=\frac{\hat{Y}-17 / 15}{\hat{Y}+2 / 5} \frac{2 \hat{Z}}{\hat{Y}-4 / 3} \frac{\hat{Z}-\hat{Z}_{N}(\hat{Y})}{\hat{Z}-\hat{Z}_{D}(\hat{Y})},
$$

together with

$$
\hat{\eta} \frac{d \hat{Y}}{d \hat{\eta}}=-\left(\hat{Y}+\frac{2}{5}\right) \frac{\hat{Z}-\hat{Z}_{D}(\hat{Y})}{\hat{Z}-\hat{Z}_{P}(\hat{Y})},
$$

where

$$
\begin{aligned}
& \hat{Z}_{P}=\frac{9}{5}\left(\hat{Y}-\frac{4}{3}\right)^{2}, \\
& \hat{Z}_{N}=\frac{9}{5} \frac{(\hat{Y}-4 / 3)(\hat{Y}-1)-\hat{Y} / 9}{\hat{Y}-17 / 15}\left(\hat{Y}-\frac{4}{3}\right), \\
& \hat{Z}_{D}=\frac{9}{5} \frac{\hat{Y}(\hat{Y}-1)}{\hat{Y}+2 / 5}\left(\hat{Y}-\frac{4}{3}\right) .
\end{aligned}
$$




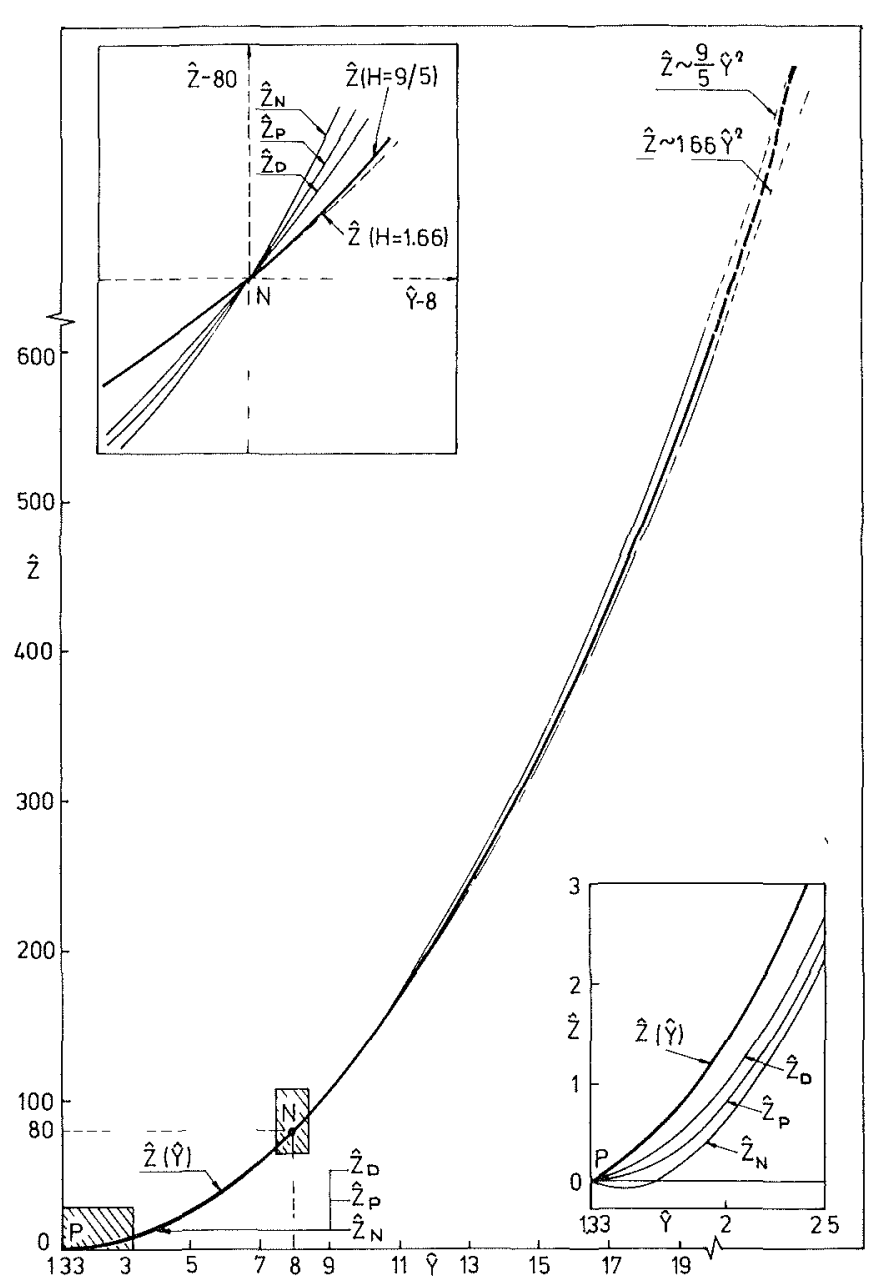

FIG. 3. Integral solution $\hat{Z}(\hat{Y})$ of Eq. (38). The inserts represent the neighborhood of the singular points $\mathrm{N}$ (schematic) and P.

Since $4 \hat{\eta}-3 \hat{y} \geqslant 0$, the solution $\hat{Z}(\hat{Y})$ to Eq. (38) must stay to the right of $\hat{Y}=4 / 3$; for $\hat{Y} \geqslant 4 / 3$, all three curves $\hat{Z}_{P}(\hat{Y}), \hat{Z}_{N}(\hat{Y})$, and $\hat{Z}_{D}(\hat{Y})$ cross each other at $\mathbf{P}$ and $\mathrm{N}$ [which are singular points of $(38)$ ] and behave as $(9 / 5) \hat{Y}^{2}$ as $\hat{Y} \rightarrow \infty$ (see Fig. 3). We also notice that in dimensional variables, $\hat{Z}=\hat{Z}_{P}(\hat{Y})$ reads

$$
\left(v-\frac{d x}{d t}\right)^{2}=\frac{5}{3} \frac{\left(Z_{i}+1\right) n k T}{m_{i} n},
$$

where $x=\frac{3}{4} v_{0} \tau(t / \tau)^{4 / 3} \xi$ is the moving plane corresponding to a given $\xi$; thus, the plasma velocity relative to this plane would be sonic on the curve $\hat{Z}_{P}(\hat{Y})$. For $\hat{Z} \gtrless \hat{Z}_{P}(\hat{Y})$, the velocity would be subsonic and supersonic, respectively; for large $\hat{Y}$, these conditions correspond to $H \gtrless 9 / 5$.

Equations (34), (35), (37), and (38) lead to the conditions

$$
\begin{aligned}
& \hat{Z} \sim H \hat{Y}^{2} \text { as } \hat{Y} \rightarrow \infty \\
& \hat{Z}=0, \quad \hat{\nu}^{7 / 3}=\frac{\hat{\eta}_{v}^{3}}{H^{2}} \frac{\hat{Z}^{2}}{\hat{Y}-4 / 3}=0, \quad \text { at } \hat{Y}=4 / 3 .
\end{aligned}
$$

Now, all solutions to Eq. (38) behave as $\hat{Z} \sim \hat{Y}^{2}$ for large $\hat{Y}$. On the other hand, solutions to Eq. (38), crossing point $\mathrm{P}$, may have any of the following three relations

$$
\hat{Z}=C(\hat{Y}-4 / 3)^{3 / 13}, \quad \hat{Z}=0, \quad \hat{Z}=\hat{Y}-4 / 3,
$$

$C$ being an arbitrary constant. However, the first two relations may be ruled out since they lead to $\hat{\nu}^{7 / 3} \neq 0$ at $\hat{Y}=4 / 3$, and $\hat{Z}=0$ everywhere, respectively. Thus, one can numerically integrate Eq. (38), starting at point $P$, along the curve $\hat{Z}=\hat{Y}-4 / 3$. The solution is then found to cross the nodal point $\mathrm{N}$ and go to infinity as $\hat{Z}=1,66 \hat{Y}^{2}$.

One should notice, nonetheless, that this solution need not be valid beyond point $N$, since there are an infinite number of solutions to Eq. (38) crossing $\mathrm{N}$ with the same first few derivatives. In fact, the analys is of the deflagration structure in Sec. $V$ proves that $H$ in (43) cannot be less than $9 / 5$; that is, the flow velocity relative to the deflagration wave, just behind it, cannot be supersonic. In addition, it can easily be shown that solutions with $H>9 / 5$ approach $\mathrm{P}$ in the form $\hat{Z}=C(\hat{Y}$ $-4 / 3)^{3 / 13}$, and may thus be excluded. Consequently, we must have $H=9 / 5$; this proves that the sonic or Chapman-Jouguet condition is satisfied here.

We find that Eq. (38) admits of two solutions with $H$ $=9 / 5$ in Eq. (43), lying to the left and right of $\hat{Z}_{p}$, respectively. The first one, however, does not reach point $\mathrm{N}$, and approaches $\mathrm{P}$ as $\hat{Z} \sim(\hat{Y}-4 / 3)^{3 / 13}$; it may also be ruled out. On the other hand, the curve to the right of $\hat{Z}_{p}$, first moves away from it and then turns and reaches point $N$. It is, then, clear that this curve, together with the solution which was previously found to go from $\mathrm{P}$ to $\mathrm{N}$, make up the function $\hat{Z}(\hat{Y})$ looked for. The entire function is shown in Fig. 3. Once $\hat{Z}(\hat{Y})$ is known, one may immediately find $\hat{y}, \hat{z}$, and $\hat{\nu}$ versus $\hat{\eta}$, numerically, from Eqs. (39), (37), and (35); they are shown in Fig. 4.

\section{THE STRUCTURE OF THE DEFLAGRATION WAVE}

The deflagration layer which separates the isentropic expansion and compression regions is governed by dissipative terms, the dominant one being electron heat conduction. Hence, the thickness of this layer can be determined by requiring that energy convection and conduction be comparable. Writing the conservation equations (18) in the variables $\hat{\eta}, \hat{y}, \hat{\nu}$, and $\hat{z}$ (allowing for different temperatures $\hat{z}_{e}$ and $\hat{z}_{i}$, since, as we shall see, the layer is too thin for temperature relaxation to be effective), we obtain

$$
\begin{aligned}
& 4 \hat{\nu}= \frac{d}{d \hat{\eta}}[\hat{\nu}(4 \hat{\eta}-3 \hat{y})] \\
& 5 \hat{\nu} \hat{y}= \frac{d}{d \hat{\eta}}\left[\hat{\nu} \hat{y}(4 \hat{\eta}-3 \hat{y})-H \hat{\nu} \frac{Z_{i} \hat{z}_{e}+\hat{z}_{i}}{Z_{i}+1}\right] \\
& 3 \hat{\nu}\left(\hat{y}^{2}+H \frac{Z_{i} \hat{z}_{e}+\hat{z}_{i}}{Z_{i}+1}\right) \\
&=\frac{d}{d \hat{\eta}}\left[\frac{\hat{\nu}}{2}(4 \hat{\eta}-3 \hat{y})\left(\hat{y}^{2}+H \frac{Z_{i} \hat{z}_{e}+\hat{z}_{i}}{Z_{i}+1}\right)-\hat{\nu} \hat{y} H \frac{Z_{i} \hat{z}_{e}+\hat{z}_{i}}{Z_{i}+1}\right. \\
&\left.+\frac{c_{1} \hat{z}_{e}^{5 / 2}}{\epsilon^{2} \alpha^{3 / 2}} \frac{d \hat{z}_{e}}{d \hat{\eta}}\right]+c_{2} \delta\left(\hat{\eta}-\hat{\eta}_{c}\right),
\end{aligned}
$$




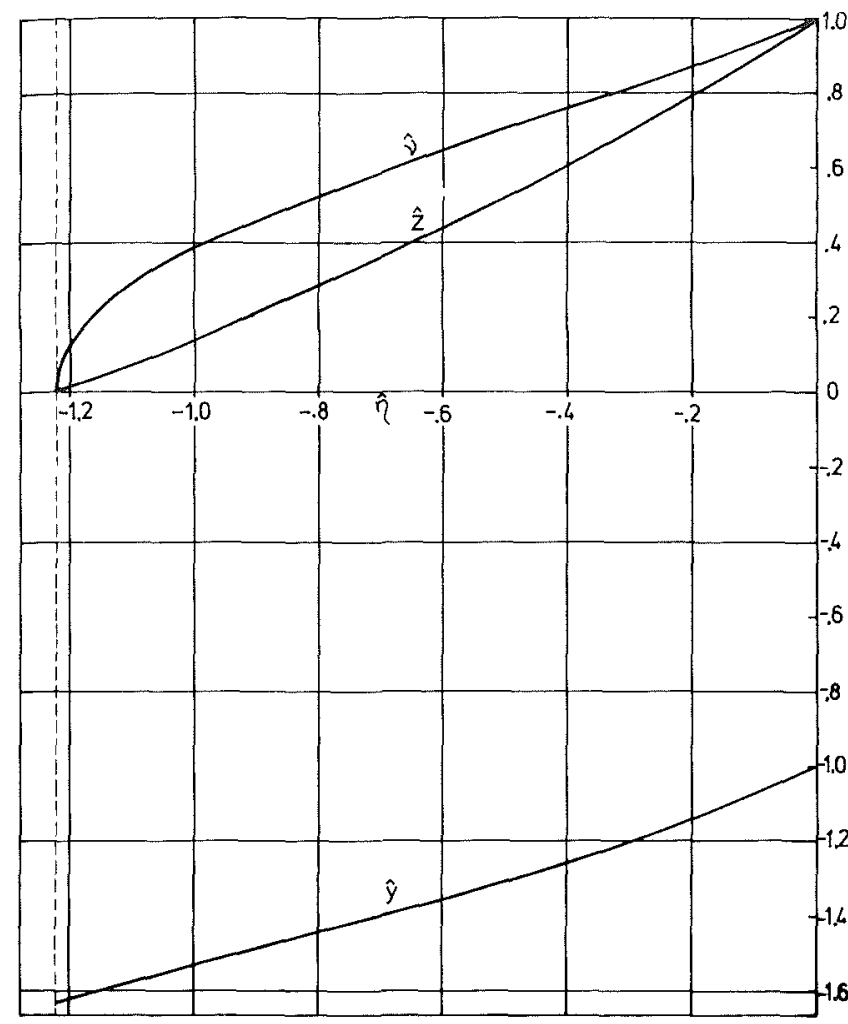

FIG. 4. Density, $\hat{v} \equiv \nu / \nu_{d}$, velocity, $\hat{y} \equiv y /\left(-y_{d}\right)$, and ion and electron temperatures $\hat{z} \equiv z / z_{d}$, vs distance, $\hat{\eta} \equiv \eta /\left(-y_{d}\right)$, within the isentropic expansion region; $\nu_{d}, z_{d}$, and $y_{d}$ are the values behind the deflagration, given in Sec. $v$.

where both $c_{1}$ and $c_{2}$

$$
c_{1} \equiv\left(\frac{3}{4}\right)^{5 / 2} \frac{Z_{i} H^{2} \bar{\gamma}^{3} z_{d}^{3 / 2} \epsilon^{5 / 2}}{4 \nu_{d}\left(Z_{i}+1\right)^{7 / 2}}, \quad c_{2} \equiv \frac{Z_{i} \epsilon^{-1 / 2}}{3 \nu_{d}\left(-y_{d}\right)^{3} \bar{\gamma}^{3}},
$$

are of order of unity. Since all four $\hat{y}, \hat{\nu}, \hat{z}_{e}$, and $\hat{z}_{i}$ must also be of order of unity, there then results a deflagration wave thickness $\hat{\eta}=O\left(\epsilon^{-2} \alpha^{-3 / 2}\right)$; it also follows that such a wave (a conduction layer, thin compared with the isentropic expansion region) will exist as long as $\alpha \gg \epsilon^{-4 / 3}$, as found before. We notice, on the other hand, that in the compression region $\hat{\eta}=O\left(\epsilon^{1 / 2}\right)$, and therefore, the deflagration will be thinner or thicker than this region, for $\alpha \gg \epsilon^{-5 / 3}$ and $\alpha \ll \epsilon^{-5 / 3}$, respectively.

We now introduce a new scaled variable

$$
\hat{\hat{\eta}}=\frac{\alpha^{3 / 2} \epsilon^{2}}{c_{1}}\left(\hat{\eta}-\frac{\bar{\eta}}{\left(-y_{d}\right)}\right)
$$

into Eqs. (45) and integrate them once, neglecting their left-hand sides. Making use of the conditions behind the deflagration $(\hat{\hat{\eta}} \rightarrow-\infty)$

$$
\hat{\nu}=\hat{z}_{e}=\hat{z}_{i}=1, \quad \hat{y}=-1,
$$

and dropping $\hat{\hat{\eta}}$ everywhere, we arrive at

$$
\begin{aligned}
& \hat{v} \hat{y}=-1, \\
& 3 \hat{y}+H\left(Z_{i} \hat{z}_{e}+\hat{z}_{i}\right)\left[\hat{y}\left(Z_{i}+1\right)\right]^{-1}=-3-H, \\
& \frac{3}{2} \hat{y}^{2}+\frac{5}{2} H\left(Z_{i} \hat{z}_{e}+\hat{z}_{i}\right)\left(Z_{i}+1\right)^{-1}+\hat{z}_{e}^{5 / 2} d \hat{z}_{e} / d \hat{\eta} \\
& \quad+c_{2} \sigma=\frac{1}{2}(3+5 H),
\end{aligned}
$$

$\sigma$ being zero if $\hat{\hat{\eta}}<\hat{\hat{\eta}}_{c}$, and unity if $\hat{\hat{\eta}}>\hat{\hat{\eta}}_{c}$. A fourth equation, to determine ion temperature, is provided by (11b)

$$
\hat{z}_{i} \frac{d \hat{y}}{d \hat{\hat{\eta}}}+\frac{3}{2} \hat{y} \frac{d \hat{z}_{i}}{d \hat{\hat{\eta}}}=\frac{3 \times 4.3 b Z_{i}^{2}}{4\left(Z_{i}+1\right)^{2}} H^{2} \hat{\nu} \frac{\hat{z}_{e}-\hat{z}_{i}}{\hat{z}_{e}^{3 / 2}} .
$$

Evaluating Eqs. (48b) and (48c) ahead of the wave $(\hat{\hat{\eta}}=0)$, where $\hat{z}_{e} / \hat{z}_{i}=1, \hat{z}_{e}$ and $\hat{y}$ vanish, and $\hat{\nu} \hat{z}_{e}=B_{1} B_{2} /$ $\nu_{d} z_{d}$, we find the jump conditions across the wave

$$
3+H=H B_{1} B_{2} / \nu_{d} z_{d}, \quad c_{2}=\frac{1}{2}(3+5 H) .
$$

As in Ref. 1, the continuity equation yields no condition, the mass flow at $\bar{\eta}$ being unknown.

System (48) yields

$\hat{v}=-1 / \hat{y}, \quad \hat{z}_{i}=-Z_{i} \hat{z}_{e}-H^{-1}\left(Z_{i}+1\right) \hat{y}(3+H+3 \hat{y})$,

together with coupled equations for $\hat{z}_{e}$ and $\hat{y}$

$\frac{d \hat{z}_{e}}{d \hat{\hat{\eta}}}=\frac{6 F(\hat{y})}{\hat{z}_{e}^{5 / 2}}, \frac{d \hat{y}}{d \hat{\hat{\eta}}}=\frac{3 \times 4.3 b H^{2} Z_{i}}{4\left(Z_{i}+1\right)} \frac{\left[\hat{z}_{e}-\hat{z}_{1}(\hat{y})\right]\left[\hat{z}_{e}-\hat{z}_{2}(\hat{y})\right]}{\hat{y} \hat{z}_{e}^{5 / 2}\left[\hat{z}_{e}-\hat{z}_{p}(\hat{y})\right]}$,

where

$$
\begin{array}{ccc}
F(\hat{y})=(\hat{y}+1)[\hat{y}+(3+5 H) / 12], & \sigma=0 \\
=\hat{y}[\hat{y}+5(3+H) / 12], & \sigma=1 \\
\hat{z}_{p}(\hat{y})=-12 \frac{Z_{i}+1}{Z_{i}} \frac{\hat{y}}{H}[\hat{y}+5(3+H) / 24], \\
\hat{z}_{1,2}(\hat{y})=-\frac{\hat{y}}{2 H}\left[H+3+3 \hat{y} \pm\left\{(H+3+3 \hat{y})^{2}\right.\right. \\
\left.\left.+\frac{48}{4.3 b} \frac{Z_{i}+1}{Z_{i}} F(\hat{y})\right\}^{1 / 2}\right] .
\end{array}
$$

Equations (51) lead to a phase-space equation for $\hat{z}_{e}$ and $\hat{y}$

$$
\frac{d \hat{z}_{e}}{d \hat{y}}=\frac{8\left(Z_{i}+1\right)}{4.3 b Z_{i} H^{2}} \frac{\hat{y} F(\hat{y})\left[\hat{z}_{e}-\hat{z}_{p}(\hat{y})\right]}{\left[\hat{z}_{e}-\hat{z}_{1}(\hat{y})\right]\left[\hat{z}_{e}-\hat{z}_{2}(\hat{y})\right]},
$$

which must be solved subject to two boundary conditions

$$
\hat{z}_{e}(\hat{y}=-1)=1, \quad \hat{z}_{e}(\hat{y}=0)=0 .
$$

We notice, however, that both $H$ and the value of $\hat{y}$ at the critical density plane $\hat{y}_{c}$ (such that $\sigma=0$ for $\hat{y}<\hat{y}_{c}$, and $\sigma=1$ for $\hat{y}>\hat{y}_{c}$ ), must be found as part of the problem.

Clearly, the solution curve $S$ will be comprised of two parts: a solution to Eq. (55) with $\sigma=1$, passing through the origin, $S_{1}(H)$, and a solution to Eq. (55) with $\sigma=0$, passing through point $(-1,1), S_{2}(H)$, both parts meeting at the critical point $\left(\hat{y}_{o}, \hat{z}_{o}\right)$. In what follows we shall prove that $S$ does not exist for $H$ less than $9 / 5$, while it does for $H=9 / 5$; since, on the other hand, the range $H>9 / 5$ was ruled out by the analysis of Sec. IV, we would then conclude that $H=9 / 5$.

We, first, find out that for $H \leqslant(9 / 5)\left(Z_{i}+1\right) /\left(1+3 Z_{i} / 5\right)$, point $(-1,1)$ lies outside the parabola $\hat{z}_{e}=\hat{z}_{p}(\hat{y})$, while $S_{1}(H)$ lies inside it for $\hat{z}_{e}$ small and positive, for all $H$. Thus, for $H \leqslant 9 / 5, \hat{z}_{e}-\hat{z}_{p}(\hat{y})$ must vanish somewhere; since $d \hat{\eta} / d \hat{y}$ should vanish nowhere (otherwise, $\hat{y}$ would not be a single-valued function of $\hat{\hat{\eta}}$ ), the cross point of $S$ and $\hat{z}_{p}$ must be an intersection of $\hat{z}_{p}$ and $\hat{z}_{1}$ (or $\hat{z}_{p}$ and $\hat{z}_{2}$ ). 


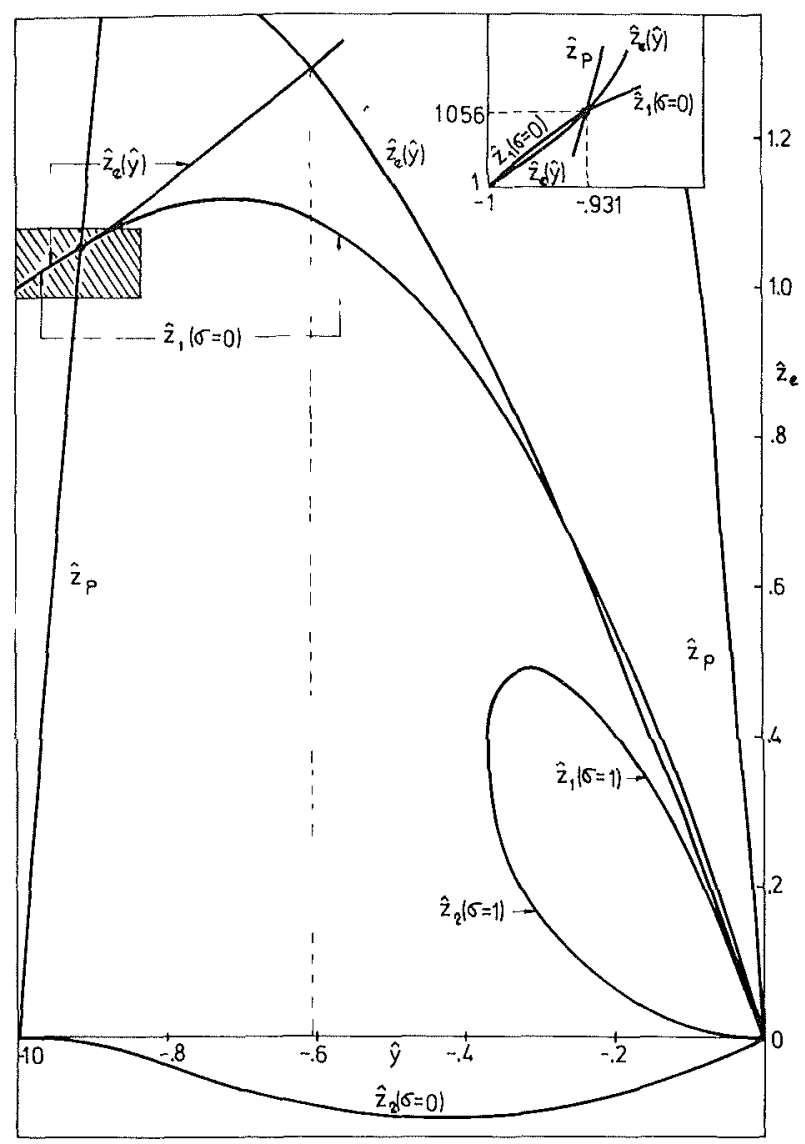

FIG. 5. Integral solution $\hat{z}_{e}(\hat{y})$ of Eq. (55) for $Z_{i}=1(H=9 / 5)$. The insert represents schematically the neighborhood of point $(-1,1)$.

We next find that $S_{1}(H)$ should reach the critical point inside the parabola. Figure 5 shows $\hat{z}_{p}, \hat{z}_{1}$, and $\hat{z}_{2}$ in the range $-1 \leqslant \hat{y} \leqslant 0$, for both $\sigma=1$ and $\sigma=0, Z_{i}=1$, and $H=9 / 5$; the parabola and the loop formed by $\hat{z}_{1}(\sigma=1)$ and $\hat{z}_{2}(\sigma=1)$, have no common point, except the origin. As $H$ decreases to zero, both the loop and the parabola get narrower and taller, but remain separate. On the other hand, as $Z_{i}$ increases, the loop becomes broader while $\hat{z}_{p}(\hat{y})$ is reduced (by as much as one half), and they finally cross each other for large enough $Z_{i}$. Nonetheless, $d \hat{z}_{1} / d \hat{y}$ is positive at the crosspoint; since $S_{1}(H)$ leaves the origin between $\hat{z}_{1}(\hat{y})$ and $\hat{z}_{p}(\hat{y})$, it cannot, clearly, reach that point.

Now, for $H<9 / 5$, point $(-1,1)$ is a saddle having a positive and a negative slope, and $\hat{z}_{1}(\sigma=0)$ lies in between. It is easy to show that $\hat{y}$ must be increasing at $(-1,1)$ and that the branch with positive slope, $S_{21}(H)$, cannot be part of $S$, since it is incompatible with Eqs. (51). To prove that the other branch, $S_{22}(H)$, also cannot be part of $S$ we consider the relative positions of the line $\hat{y}=-(3+5 H) / 12$, where $F(\hat{y}, \sigma=0)$ vanishes, and the point $M$ where $\hat{z}_{p}(\hat{y})$ and $\hat{z}_{1}(\hat{y}, \sigma=0)$ meet. Let $H^{*}\left(Z_{i}\right)$ be the value of $H$ for which $M$ lies on that line ( $H^{*}$ ranges from $27 / 19$ at $Z_{i}=1$, to unity for $Z_{i}$ infinite). For $H$ $<H^{*}$, M lies to the left of the line, so that the slope of $S_{22}$ must remain negative until crossing either $\hat{z}_{p}$ or $\hat{z}_{1}$; since it can be shown that $\hat{z}_{p}>1$ at $\mathrm{M}, S_{22}(H)$ cannot be part of $S$. Actually, for $H$ small enough $\hat{z}_{1}(\sigma=0)$ and $\hat{z}_{2}(\sigma=0)$ form two loops, one starting at the origin, the other crossing both $(-1,1)$ and $(-1,0)$; then, $S_{22}(H)$ is trapped inside this second loop and finally crosses $\hat{z}_{2}(\sigma=0)$ toward decreasing $\hat{y}$. For $9 / 5>H>H^{*}$, M lies to the right of $\hat{y}=-(3+5 H) / 12$, which meets $\hat{z}_{1}(\sigma=0)$ at a point which is a node of $\mathrm{Eq} .(55)$, while $\mathrm{M}$ is a saddle. We find that $S_{22}(H)$ does not reach $M$. Thus, $S$ does not exist for $H<9 / 5$. We notice that, actually, a negative slope at $(-1,1)$ would imply that heat would flow toward the plane (far to the left) where energy is absorbed.

We find, numerically, that for $Z_{i}$ not too large, $S_{21}(H<9 / 5)$ crosses the node from above $\hat{z}_{1}(\sigma=0)$ and reaches $M$ from below $\hat{z}_{1}(\sigma=0)$. As $H$ goes to $9 / 5$, the node moves to point $(-1,1)$; then, both $S_{21}$ and $S_{22}$ leave this point below $\hat{z}_{1}(\sigma=0)$ and are thus compatible with Eqs. (51). Since clearly $S_{21}$ still reaches $M, H$ must be $9 / 5$, and the solution $S$ will be composed of $S_{1}(9 / 5)$ and $S_{21}(9 / 5)$, which should meet inside the parabola, determining $\hat{y}_{c}$. We notice, that as $Z_{i}$ increases, the critical point approaches the parabola, reaching it at $Z_{i}=\infty$.

The entire solution is shown in Fig. 5 for $Z_{i}=1$. Once $H$ and $\hat{y}_{c}$ are known, Eq. (48a) leads to $\nu_{d}$

$$
-\frac{1}{\hat{y}_{0}}=\hat{\nu}_{c}=\frac{\nu_{c}}{\nu_{d}}=\frac{\epsilon}{4 \nu_{d}} \text {. }
$$

Then, $z_{d}$ and $c_{2}$ can be obtained from (49). Finally, using $y_{d}=\left(5 z_{d} / 9\right)^{1 / 2}$, both $\bar{\gamma}$ and the scale constant $c_{1}$ are given by Eqs. (46). All $\hat{y}, \hat{z}_{e}, \hat{z}_{i}$, and $\hat{\nu}$ are shown in Fig. 6 as functions of $\hat{\hat{\eta}}$, for $Z_{i}=1$. Table I gives $n_{d}\left(\equiv 4 n_{0} \nu_{d}\right), T_{e c}$ (maximum $T_{e}$, at the critical plane), $c_{1}$, and $\xi_{f}\left(\equiv \bar{\gamma} \epsilon^{1 / 6} \alpha^{1 / 3}\right)$, for several values of $Z_{i}$, to complete the solution for the isentropic expansion. Notice that the sonic or Chapman-Jouguet condition $(H=9 / 5)$ and the value $\nu_{d}$ follow from the analysis of the deflagration structure, independently of the results for the compression and expansion flows. Then, the momentum and energy conservation equations (49), written in dimensional form yield

$$
P_{a}=\frac{8 P_{d}}{3}, \quad P_{d}=\frac{3}{5}\left(\frac{m_{i} n_{d} \phi^{2}}{4 Z_{i}}\right)^{1 / 3},
$$

(the subscript $a$ refers to conditions ahead of the deflagration) while

$$
T_{d}=Z_{i} p_{d} /\left(1+Z_{i}\right) k n_{d}, \quad v_{d}^{2}=5\left(1+Z_{i}\right) k T_{d} / 3 m_{i}
$$

(the velocity of the deflagration front is negligible compared with $v_{d}$ ). Consequently, the results for both the deflagration layer and expansion, together with $P_{a}$ and the mass flux into the deflagration, are independent of the behavior in the compression region.

\section{DISCUSSION OF RESULTS}

We have studied the one-dimensional self-similar motion of an initially cold, half-space plasma of electron density $n_{0}$, when a laser pulse $\phi=\phi_{0} t / \tau(t \leqslant \tau)$ is (anomalously) absorbed at the critical density $n_{c}$ (its location being part of the solution). We allowed heat conduction and different temperatures $T_{e}$ and $T_{i}$. The solution is governed by three dimensionless numbers: ion charge 


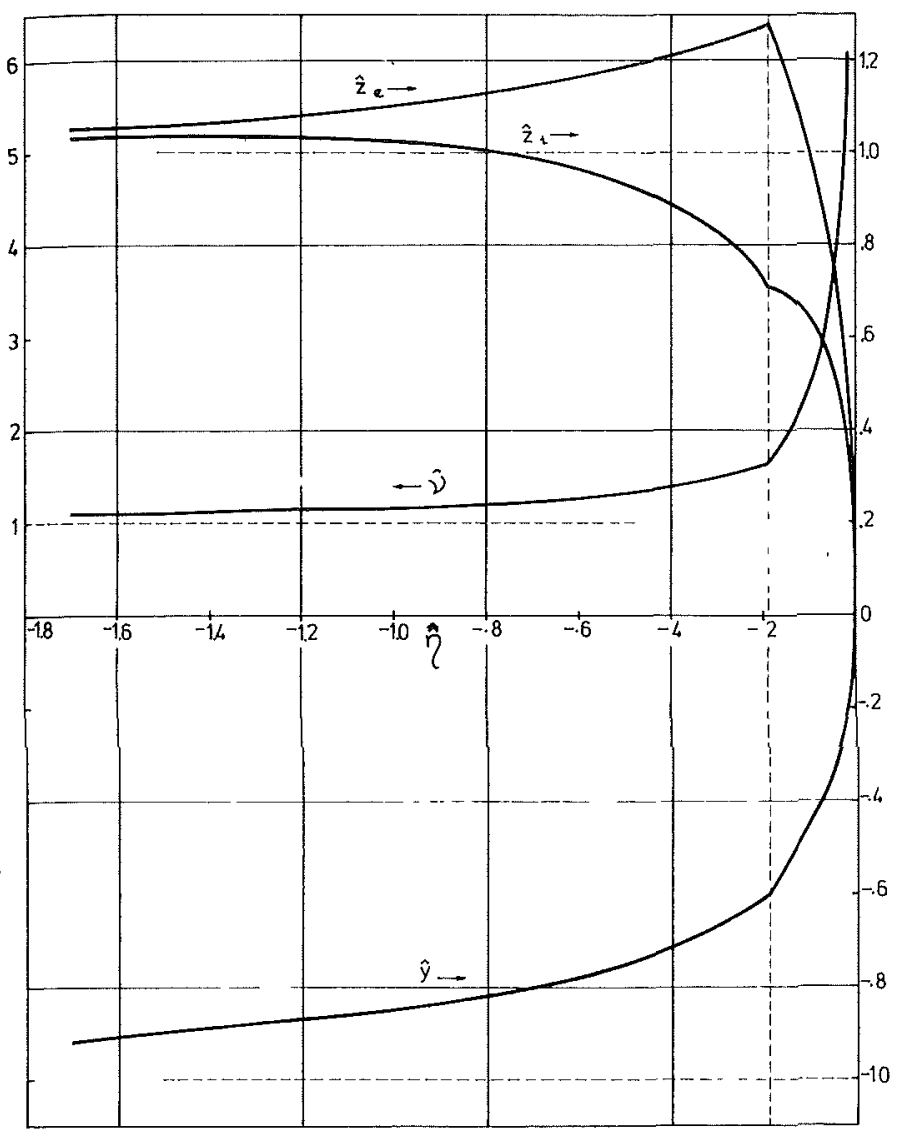

FIG. 6. Density, $\hat{\nu} \equiv \nu / \nu_{d}$, velocity, $\hat{y} \equiv y /\left(-y_{d}\right)$, and electron and ion temperatures, $\hat{z_{j}} \equiv z_{j} / z_{d}$, vs distance, $\hat{\hat{\eta}} \equiv\left(\alpha^{3 / 2} \epsilon^{2} / c_{1}\right)$ $(\eta-\bar{\eta}) /\left(-y_{d}\right)$, within the deflagration layer, for $z_{i}=1 ; \bar{\eta}=0.82$, $c_{1}$ given in Table I.

number $Z_{i}$, density ratio $\epsilon \equiv n_{c} / n_{0}$ (small), and a parameter $\alpha$, defined in Eq. (8). Here we considered the regime $\alpha \gg \epsilon^{-4 / 3}$, or more precisely, $\alpha^{3 / 2} \epsilon^{2} \gg 1$, which is given in $\mathrm{Eq}$. (1)

$$
2.10 \times 10^{-30} \frac{Z_{i}}{b\left(Z_{i}\right)} \frac{\tau(\mathrm{nsec})\left[n_{c}\left(\mathrm{~cm}^{-3}\right)\right]^{2}}{A_{i}^{3 / 2} \phi_{0}\left(\mathrm{~W} \mathrm{~cm}^{-2}\right)} \gg 1,
$$

[ $A_{i}$ is the ion mass number, $b=O(1)$ given in Eq. (2)]; $Z_{i}$ is left arbitrary. Then, we find that a deflagration wave (inside which energy absorption occurs, $T_{e} \neq T_{i}$, and heat conduction is important) separates an isentropic compression flow, bounded by a shock from the undisturbed plasma, and an isentropic expansion flow bounded by the vacuum. We determined the solution throughout the entire plasma. The criterion in Eq. (1) can be extended to general pulses $\phi(t)$, for which a characteristic value of $\phi(t) / t$ or $d \phi / d t$ (whichever is the larger) may be reasonably defined to use in (1) instead of $\phi_{0} / \tau$.

The compression to expansion ratio for both energy and thickness is of the order of $\epsilon^{1 / 2}$. The width of the deflagration layer is

$$
\Delta x(\mathrm{~cm})=6.8 \times 10^{30} \frac{b c_{1} A_{i}^{7 / 6}}{Z_{i}^{2 / 3}\left(n_{d} / n_{c}\right)^{1 / 3}} \frac{\left[\phi\left(\mathrm{W} \mathrm{cm}^{-2}\right)\right]^{4 / 3}}{\left[n_{c}\left(\mathrm{~cm}^{-3}\right)\right]^{7 / 3}},
$$

where $c_{1}$ and $n_{d} / n_{c}$ are given in Table $I$ as functions of $Z_{i}$. The deflagration is thinner (thicker) than the com- pression region for $\alpha \gg \epsilon^{-5 / 3}\left(\alpha \ll \epsilon^{-5 / 3}\right)$. For $\alpha$ small enough $\left(\alpha \sim \epsilon^{-4 / 3}\right)$ deflagration and expansion merge. Notice, however, that if $Z_{i}$ is left to go to infinity (for given $\epsilon$ and $\alpha$ ) the deflagration to expansion thickness ratio vanishes as $Z_{i}^{-3 / 2}$; consequently, a deflagration may exist (deflagration regime) even if $\alpha \sim \epsilon^{-4 / 3}$.

By determining the structure of the deflagration layer we found the density just behind it, $n_{d}$ (see Table I), and showed that the flow velocity is sonic there (Chapman Jouguet condition together with negligible wave speed). The jump conditions across the wave then give the pressure ahead (maximum pressure in the plasma)

$$
P_{a}=\frac{8}{5}\left(\frac{m_{i} n_{d} \phi^{2}}{4 Z_{i}}\right)^{1 / 3},
$$

and behind $P_{d}=3 P_{a} / 8$, together with the mass flux into the wave. We emphasize that the validity of the results for the deflagration structure and jump conditions is not limited to linear pulses and one-dimensional geometries.

We found that the deflagration to shock speed ratio is 0.82 , the shock speed in $\mathrm{cm} / \mathrm{sec}$ being $3.2 \times 10^{10} \bar{\gamma}\left(Z_{i}\right)$ $\times \epsilon^{1 / 6} A_{i}^{-1 / 3}\left(\phi / n_{0}\right)^{1 / 3}$, where $\phi$ and $n_{0}$ are measured in $\mathrm{W} \mathrm{cm} \mathrm{cm}^{-2}$ and $\mathrm{cm}^{-3}$, respectively, and $\bar{\gamma} \equiv \xi_{f} \alpha^{-1 / 3} \epsilon^{-1 / 6}\left(\xi_{f}\right.$ given in Table I). The results for the compression region have been obtained assuming that the medium, there, is a plasma; it was shown, however, that those results are also valid if the medium is a monatomic ideal gas or a condensed hydrogen isotope at pressures around $1 \mathrm{Mbar}$ and densities not far above solid density (conditions found in many situations of interest). Moreover, results for the expansion region are entirely independent of the compression flow.

We have assumed throughout the analysis that the plasma was dominated by collisions and quasi-neutral. It is easy to show, using self-similar variables, that for all regions within the plasma the ratio of mean-freepath to characteristic length is small [of order $\left(m_{e} / m_{i}\right)^{1 / 2}$ in the worst case, the deflagration layer]. The quasineutrality condition is also most critical for the deflagration, for which it reads

$$
10^{25} \phi\left(\mathrm{W} \mathrm{cm}^{-2}\right) \gg 3.5 Z_{i}\left[A_{i} b\left(Z_{i}\right)\right]^{-1}\left[n_{c}\left(\mathrm{~cm}^{-3}\right)\right]^{3 / 2} ;
$$

clearly, this inequality is easily satisfied except at the very beginning of the pulse. Because the expansion is isentropic, both Debye length and mean-free-path vanish

TABLE I. Values of the density ratio $n_{d} / n_{c},\left(n_{c}\right.$, critical density), temperature ratio $T_{e c} / T_{d}\left(T_{e c}=\right.$ electron temperature at critical plane), shock location parameter $\xi_{f}$, and deflagration width constant $c_{1}$ [Eq. (47)], for several values of $Z_{i}$. The subscript $d$ labels conditions behind the deflagration.

\begin{tabular}{ccccc}
\hline \hline$Z_{i}$ & $n_{d} / n_{c}$ & $T_{e c} / T_{d}$ & $\xi_{f} \epsilon^{-1 / 6} \alpha^{-1 / 3}$ & $c_{1}$ \\
\hline 1 & 0.61 & 1.28 & 0.53 & 0.20 \\
2 & 0.66 & 1.17 & 0.68 & 0.17 \\
4 & 0.74 & 1.13 & 0.87 & 0.09 \\
16 & 0.78 & 1.08 & 1.40 & 0.02 \\
$\infty$ & 0.80 & 1.07 & $\sim 0.47 Z_{i}^{1 / 3}$ & $\sim 0.80 Z_{i}^{-3 / 2}$ \\
\hline
\end{tabular}


at the vacuum-plasma front; thus, the charge separation effect found by Crow et al. ${ }^{12}$ for isothermal expansions, for which the Debye length and mean-free-path become infinite, does not apply here. Notice, finally, that the approximations used for the Coulomb logarithms in the heat conductivity and the energy exchange relaxation time, affect only the structure of the deflagration (where they enter as a ratio) and its thickness.

\section{ACKNOWLEDGMENT}

This research was performed under the auspices of the Junta de Energia Nuclear of Spain.

\section{APPENDIX}

To analyze the deflagration, we assumed $\hat{y}(\hat{\hat{\eta}}=0)=0$. Actually, for matching with the isentropic compression solution, we should have $y(\eta=\bar{\eta})=4 \bar{\eta} / 3$, that is, $y(\hat{\hat{\eta}}=0)$ $=4 \bar{\eta} / 3\left(-y_{d}\right)=O\left(\epsilon^{1 / 2}\right)$. Then, for $\hat{\hat{\eta}} \rightarrow 0$, we obtain

$$
\begin{aligned}
& z_{i} \simeq z_{e} \simeq z_{d}(45 / 4)^{2 / 5}(-\hat{\hat{\eta}})^{2 / 5}, \\
& \nu \simeq B_{1} B_{2} / z_{e}, \quad y \simeq 4 \bar{\eta} / 3+\left(3 y_{d} / 8\right) z_{e} / z_{d},
\end{aligned}
$$

which are just the deflagxation results, except for the shift in $y$.

Then, to study the transition layer at $\bar{\eta}$, we define $J \equiv \nu(4 \eta-3 y)$ and, using Eqs. (A1), find

$$
J\left(\eta \rightarrow \bar{\eta}^{-}\right) \rightarrow-9 B_{1} B_{2} y_{d} / 8 z_{d}=O\left(\epsilon^{1 / 2}\right) .
$$

On the other hand, Eqs. (25) for the isentropic compres. sion give $J=\left(26 B_{2} / 5\right)(\eta-\bar{\eta})^{10 / 13}$, which is $O\left(\epsilon^{1 / 2}\right)$ for $\eta_{1}$ such that

$$
\eta_{1}-\bar{\eta}=O\left(\epsilon^{13 / 20}\right)
$$

and hence $z=O\left(\epsilon^{3 / 20}\right), \nu=O\left(\epsilon^{-3 / 20}\right)$. Finally, to retain heat conduction we expand the variables in a thin layer of width

$$
\Delta \eta=O\left(\epsilon^{3 / 8} \alpha^{-3 / 2}\right) \ll \eta_{1}-\bar{\eta},
$$

centered at $\eta_{1}$. The analys is then proceeds as in Appendix B of Ref. 1, and yields $\eta_{1}, z_{\mathrm{min}}$, and $\nu_{\max }$; for $Z_{i}=1, \eta_{1}=\bar{\eta}+0.086 \epsilon^{13 / 20}, z_{\min }=0.965 \epsilon^{3 / 20}, \nu_{\max }$ $=1.37 \epsilon^{-3 / 20}$.

${ }^{1}$ A. Barrero and J. R. Sanmartín, Phys. Fluids 20, 1155 (1977).

${ }^{2}$ S. I. Anisimov, Zh. Eksp. Teor. Fiz. Pis'ma Red. 12, 414 (1970) [JETP Lett. 12, 287 (1970)]; see also R. Marshak, Phys, Fluids 1, 24 (1958).

${ }^{3}$ S. H. Choi and H. E. Wilhelm, Phys. Rev. A 14, 1825 (1976).

${ }^{4} \mathrm{~J}$. Nuckolls, L. Wood, A. Thiessen, and G. Zimmerman, Nature 239, 139 (1972).

${ }^{5}$ K. A. Brueckner and S. Jorna, Rev. Mod. Phys. 46, 325 (1974).

${ }^{6} J$. R. Sanmartín and A. Barrero, Phys. Fluids 21, 1967 (1978).

${ }^{7}$ C. Fauquignon and F. Floux, Phys. Fluids 13, 386 (1970).

${ }^{8} \mathrm{~J}$. L. Bobin, Phys. Fluids 14, 2341 (1971).

${ }^{9}$ G. J. Pert, Plasma Phys. 16, 1035 (1974).

${ }^{10}$ S. J. Gitomer, R. L. Morse, and B. S. Newberger, Phys. Fluids 20, 234 (1977).

${ }^{11}$ A. V. Gurevitch, L. V. Pariiskay, and L. P. Pitaevskii, Zh. Eksp. Teor. Fiz. 49, 647 (1965) [Sov. Phys, -JETP 22, 449 (1966)].

${ }^{12} \mathrm{~J}$. E. Crow, O. L. Auer, and J. E. Allen, J. Plasma Phys. 14,65 (1975).

${ }^{13}$ L. Spitzer, Physics of Fully Ionized Gases (Wiley, New York, 1962).

${ }^{14} \mathrm{P}$. Mulser, Z. Naturforsch. A 25, 282 (1970).

${ }^{15}$ R. S. Cooper, AIAA J. 11, 831 (1973).

${ }^{16}$ R. J. Mason and R. L. Morse, Phys. Fluids 1\&, 814 (1975).

${ }^{17} \mathrm{D}$. G. Colombant, K. G. Whitney, D. A. Tidman, N. K. Winsor, and J. Davis, Phys. Fluids 18, 1687 (1975).

${ }^{18}$ G. I. Kerley, Los Alamos Scientific Laboratory Report LA4776 (1972).

${ }^{19}$ J. W. Stewart, Phys. Rev. 97, 578 (1955).

${ }^{20} \mathrm{~L}$. I. Sedov, Similarity and Dimensional Methods in Mechanics (Academic, New York, 1959), Chap. IV. 